\title{
cmaJOPEN
}

\section{Coverage for pertussis vaccination during pregnancy with 4 models of vaccine delivery: a quasiexperimental, multicentre observational study}

\author{
Yinan Li HBSc MScPH, Nicholas Brousseau MD MSc, Maryse Guay MD MSc, Ève Dubé PhD, \\ Zineb Laghdir MSc, Isabelle Boucoiran MD MSc, Bruce Tapiéro MD, Caroline Quach MD MSc
}

Abstract

Background: Vaccination of pregnant people with a vaccine containing acellular pertussis (tetanus-diphtheria-acellular pertussis [Tdap]) has been recommended in Canada since 2018, and the evaluation of delivery models for efficient maternal Tdap administration is a priority for the Quebec Ministry of Health. We implemented 3 vaccine delivery models, in addition to the existing standard of practice model, and compared the vaccine coverage achieved by the 4 models in Quebec.

Methods: In this quasiexperimental, multicentre observational study, we recruited pregnant people at less than 21 weeks' gestation in 4 Quebec regions from April to October 2019. We compared 4 vaccine delivery models: local community service centres (centre local de services communautaires [CLSCs], baseline), family medicine groups (FMGs), obstetrics clinic and the oral glucose challenge test (OGCT). In addition to the CLSCs, 3 FMGs, 1 obstetric clinic and a hospital-based OGCT screening program participated. We determined vaccination status from a self-reported questionnaire, the Quebec Immunization Registry or medical charts. We compared model-specific (for participants recruited to a model and subsequently vaccinated within that model) and overall vaccine coverage (considering all vaccine delivery pathways) and used logistic regression to adjust for sociodemographic variables.

Results: Overall, 946 of 1000 recruited pregnant people were eligible for analyses. Vaccination via the FMGs achieved the highest model-specific vaccine coverage $(67.8 \%$, 95\% confidence interval $[\mathrm{Cl}] 60.5 \%-74.4 \%)$, but coverage was not significantly different from the CLSCs (63.8\%, 95\% Cl 57.6\%-69.6\%). For overall vaccine coverage, the FMG (86.5\%, 95\% Cl $80.6 \%-90.9 \%)$ and obstetrics models $(85.9 \%, 95 \% \mathrm{Cl} 80.9 \%-89.7 \%)$ achieved significantly higher vaccine coverage than the $\mathrm{CLSCs}(66.3 \%, 95 \% \mathrm{Cl} 60.1 \%-$ $71.9 \%)$. The OGCT model did not improve overall vaccine coverage $(61.8 \%, 95 \% \mathrm{Cl} 56.1 \%-67.2 \%)$.

Interpretation: Compared with CLSCs, overall vaccine coverage was higher when Tdap was offered in FMGs or an obstetrics clinic providing prenatal care. Health professionals involved in pregnancy follow-up recommending and offering the vaccine may be a key factor in optimizing vaccine coverage.

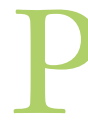

ertussis infection and complications can be severe in young infants. ${ }^{1,2}$ From 2006 to 2015 , infants younger than 1 year had the highest hospitalization rates (33.6 cases per 100000 population) in Canada. ${ }^{1}$ Infants younger than 2 months, who have not started their primary vaccination against pertussis, accounted for nearly half $(40.5 \%)$ of admissions to special care units. ${ }^{1}$ Administration of the tetanus-diphtheria-acellular pertussis (Tdap) vaccine during pregnancy increases transplacental transfer of maternal antibodies and provides direct protection of the infants. ${ }^{1,3-5} \mathrm{In}$ 2018, the National Advisory Committee on Immunization recommended the systematic vaccination of all pregnant people against pertussis, at every pregnancy. ${ }^{4}$ As of May 2018, the province of Quebec has recommended maternal Tdap immunization, ideally between 26 and 32 weeks' gestation, for every pregnancy. ${ }^{6}$ Many countries, including the United States, ${ }^{7-11}$ the United Kingdom, ${ }^{12}$ Belgium, ${ }^{13,14}$ New Zealand ${ }^{15,16}$ and Switzerland, ${ }^{17}$ have also recommended routine maternal Tdap immunization at every pregnancy. Maternal Tdap vaccine coverage has been suboptimal in several countries, ${ }^{7-14,16-20}$ such as in the US, where vaccine coverage was about $57 \%$ in 2019 2020. ${ }^{11}$ Public Health England reported that, in 2019-2020, maternal Tdap vaccine coverage was about $70 \% .{ }^{12}$ The

Competing interests: Bruce Tapiéro reports research grants from Merck, GlaxoSmithKline and Pfizer. No other competing interests were declared.

This article has been peer reviewed.

Correspondence to: Caroline Quach, c.quach@umontreal.ca

CMAJ Open 2022 February 1. DOI:10.9778/cmajo.20210011 
modalities of Tdap vaccination implementation are major factors influencing vaccine coverage. . $^{13,18,20,21}$

In Quebec, a maternal pertussis immunization program has not yet been integrated into existing medical care. A previous study, conducted in Quebec, identified challenges in implementing maternal Tdap vaccination successfully. ${ }^{21}$ Vaccination is not usually available in clinics offering prenatal care and is mainly offered in local public health clinics (local community service centres [centre local de services communautaires], CLSCs) by appointment. However, the study did not determine a specific model for efficient Tdap delivery. ${ }^{21}$ Other studies showed that Tdap vaccine coverage improved when vaccination was offered in traditional prenatal care settings by general practitioners or in obstetrics clinics., ${ }^{9} 12$ Gestational diabetes screening was also identified as a possible vaccination venue, but has not been well studied. ${ }^{21-23}$

As a follow-up to the previous study,,$^{21}$ we aimed to assess and compare the vaccine coverage associated with 4 provincebased implementation models of maternal Tdap vaccine delivery (1 existing and 3 implemented models), namely vaccination from CLSCs, family medicine groups (FMGs), obstetrics clinic and at time of screening with the oral glucose challenge test (OGCT). In this study, we evaluated vaccine coverage as 2 measures, model-specific and overall. The model-specific vaccine coverage is the coverage achieved according to the care pathway defined in the model. This measure reflects vaccine implementation and will help inform resource allocation and use. The overall vaccine coverage is the coverage achieved considering all doses, regardless of the care pathway, which assesses the perceived importance of vaccination recommendation by health care professionals.

\section{Methods}

\section{Study design and setting}

We used a quasiexperimental, multicentre study design with nonequivalent control groups of pregnant people. We recruited participants from 4 health regions of Quebec, Montréal (urban city with about 2070000 inhabitants), Montérégie (second most populated region of urban and rural areas, with about 1600000 inhabitants), Capitale-Nationale (Québec and other areas, with about 760000 inhabitants) and Mauricie (urban and rural areas, with about 270000 inhabitants)..$^{24,25}$

\section{Participants}

From April to October 2019, we recruited pregnant people at less than 21 weeks' gestation in the 4 Quebec health regions. We excluded participants if they were younger than 18 years, spoke neither French nor English, provided an invalid email address, had a miscarriage or moved out of Quebec during the pregnancy. Trained administrative personnel recruited participants during their first medical visit where their pregnancy was followed (Montérégie, Capitale-Nationale and Mauricie); in Montréal, a research assistant recruited participants during their 12-week appointment for laboratory tests and nuchal clarity ultrasonography.

\section{Vaccine delivery models}

Four models of vaccine delivery were implemented in the 4 Quebec health regions where participants were recruited. Vaccine administration ran from May 2019 to March 2020.

\section{Local community service centres model}

In Montérégie, a nurse recruited participants at an FMG clinic and referred them to their CLSC for vaccine administration. The CLSCs offer community health care services and serve a specific geographical area. ${ }^{21}$ This model is the current standard for Tdap vaccination in Quebec and served as the baseline model. ${ }^{21}$ This study component was managed by M.G.

\section{Family medicine group model}

In Montérégie and Capitale-Nationale, participants were offered Tdap vaccination at the FMG where their pregnancy was followed by a team of family physicians and nurses, on the day it was recommended. Three FMGs served as the recruitment and vaccination sites for this model, with each following 200-400 pregnancies annually. This study component was managed by M.G. and N.B.

\section{Obstetrics clinic model}

In Mauricie, participants received Tdap vaccination in a highspeed obstetrics follow-up clinic, where about 1200 pregnancies are followed annually. The obstetrician recommended Tdap at the obstetrical appointment and a nurse offered the vaccine at a separately scheduled nursing appointment in the same clinic. This study component was managed by N.B.

\section{Oral glucose challenge test model}

In Montréal, when participants were screened for gestational diabetes between weeks 24 and 28 of gestation through an OGCT (50-75 g of glucose) at a mother and child teaching hospital, they were offered Tdap vaccination during the wait hour of the same appointment or after blood procurement. Pregnant people with preexisting diabetes were referred to CLSCs for vaccination. This study component was managed by C.Q.

\section{Variables}

We collected sociodemographic and contact information through a paper questionnaire at recruitment. At week 35 of pregnancy, participants completed an online questionnaire on self-reported vaccination status, date and place of vaccination. Vaccination records from the Quebec Immunization Registry were extracted by the Quebec Ministry of Health and reviewed (Y.L.) to validate vaccination status. When possible, we (N.B., M.G. and C.Q.) reviewed medical charts to validate vaccination status for participants who indicated unknown vaccination status or did not answer the questionnaire, and for whom a proof of vaccination could not be found in the provincial registry. We reviewed medical charts for all models, except for the CLSC model, given logistical constraints. We considered a participant to be vaccinated if they self-reported as vaccinated in the online questionnaire, if they provided a vaccination date and nonvaccination was not documented in 
the medical chart, or when there was proof of vaccination in the registry or the medical chart.

\section{Outcomes}

We computed the primary outcome, model-specific vaccine coverage, as the proportion of participants who received Tdap vaccination according to the specific vaccine delivery pathway defined by the model, out of all those eligible for each model (x 100). We computed the secondary outcome, overall vaccine coverage, as the proportion of participants vaccinated with Tdap, considering all vaccine delivery pathways out of all eligible participants for each model $(\times 100)$.

\section{Statistical analysis}

We calculated the required sample size to be 162 per vaccine delivery model to detect a $15 \%$ difference in vaccine coverage between models (55\% v. 70\%) with an $\alpha$ of $5 \%$ and power of $80 \%$.

We compared vaccine coverage for each model to the baseline model using the $\chi^{2}$ test ( $\alpha=5 \%, 2$-sided tests). We used multivariable logistic regression to adjust for sociodemographic characteristics and to compute adjusted odds ratios (ORs) of vaccination for each model. We performed all analyses using $\mathrm{R}$ version 4.0.3.

We conducted sensitivity analyses based on different assumptions for participants without self-reported vaccination status and immunization records in the CLSC model, and for participants with diabetes or prediabetes who did not attend the OGCT, as well as vaccine coverage calculated using aggregated data collected for the OGCT model (Appendix 1, Supplementary Methods, available at www.cmajopen.ca/content/10/1/E56/ suppl/DC1).

\section{Ethics approval}

The Centre hospitalier universitaire Sainte-Justine research ethics board reviewed the study protocol and approved the research project.

\section{Results}

Overall, 946 of 1000 recruited participants were eligible for analyses (Figure 1). The median maternal age was 31 years. A large proportion of participants were born in Canada $(n=674$, $71.2 \%)$, Francophone $(n=755,79.8 \%)$ and married $(n=842$, $89.0 \%)$. About half had university-level education $(n=505$, $53.4 \%)$ and were in their first pregnancy $(n=411,43.5 \%)$. Participants significantly differed in sociodemographic characteristics across models (Table 1), with the OGCT model having more non-Francophones, more participants born outside of Canada and more university graduates. For the FMG model, consisting of 3 recruitment sites, characteristics by site are summarized in Appendix 1, Table S1.

\section{Vaccination status}

We obtained questionnaire response on vaccination status for $619(65.4 \%)$ of 946 participants, and $503(81.3 \%)$ of them self-reported as vaccinated. Using the Quebec Immunization
Registry, we found proof of vaccination for 174 additional participants, including 4 who self-reported as nonvaccinated in the questionnaire. Furthermore, we found 23 additional doses by reviewing medical charts. We identified proof of nonvaccination for 4 participants in the OGCT model who self-reported as vaccinated.

Overall, 696 (73.6\%) of 946 participants received a Tdap vaccine during their pregnancy (Figure 2).

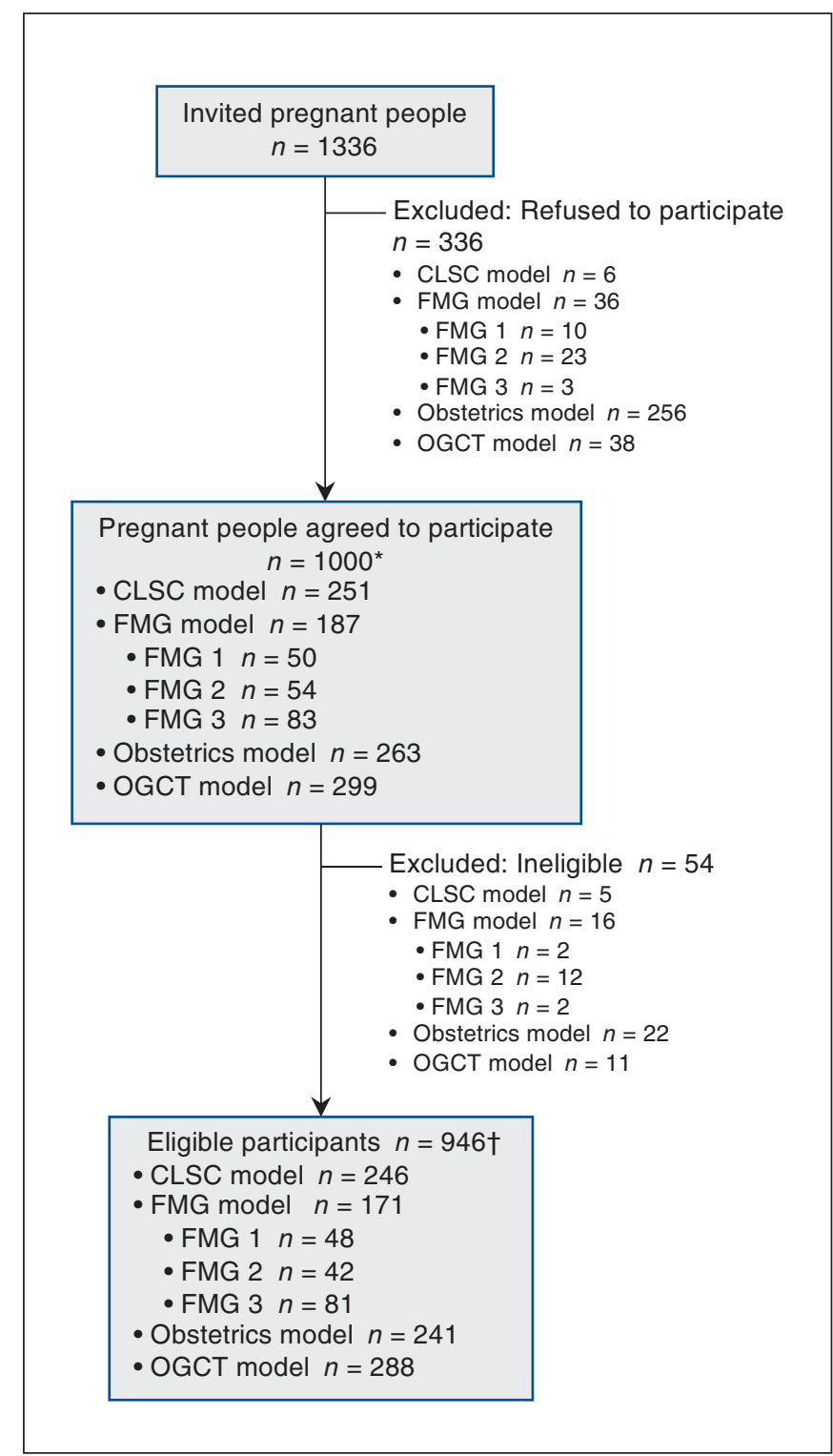

Figure 1: Participant recruitment and eligibility. Note: CLSC = local community service centre, FMG = family medicine group, OGCT $=$ oral glucose challenge test. Participation rate by model: CLSC model, 251/257 (97.7\%); FMG model, overall: 187/223 (83.9\%), FMG 1: 50/60 (83.3\%), FMG 2: 54/77 (70.1\%), FMG 3: 83/86 (96.5\%); obstetrics model, $263 / 519$ (50.7\%); OGCT model, 299/337 (88.7\%). ${ }^{*}$ Overall participation rate: 1000/1336 (74.9\%). †Overall, 946 pregnant people were eligible after the exclusion of 54 pregnant people (14 based on age or gestational age, 16 who miscarried, 3 who later refused to participate, 9 with duplicate information, 11 with missing information and 1 no longer residing in Quebec). 


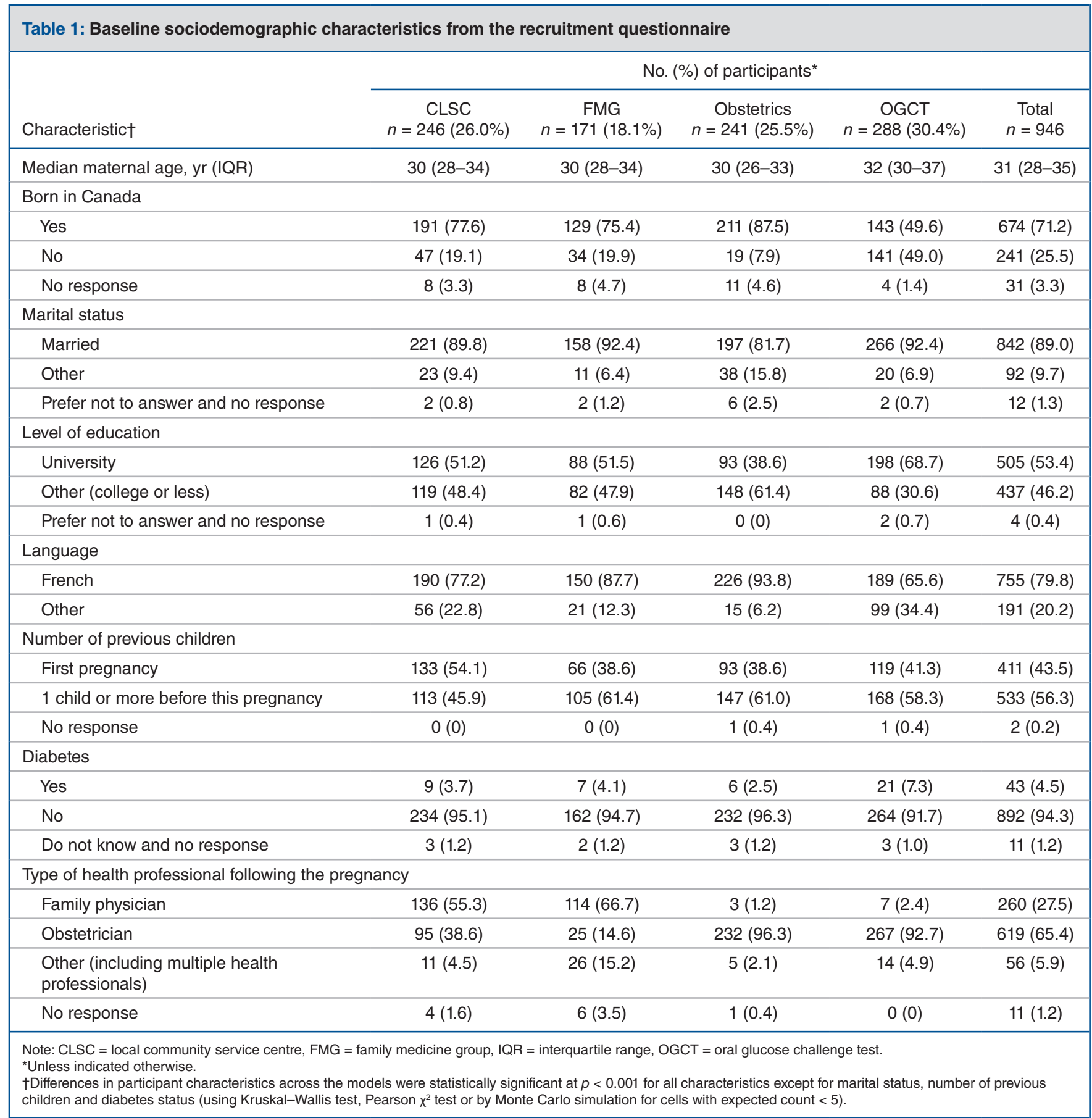

\section{Model-specific vaccine coverage}

To compare model performance, we evaluated modelspecific vaccine coverage, considering only doses delivered within each model. For the baseline CLSC model, $96.3 \%$ of participants recruited to this model who had received their Tdap did so within the CLSC vaccine delivery model (referral to the nearest CLSC). The CLSC model-specific vaccine coverage was $63.8 \%$ (95\% confidence interval [CI] 57.6\%$69.6 \%$ ) (Table 2 and Figure 3). In comparison, under the FMG model, $78.4 \%$ of vaccinated participants recruited to the FMG model were vaccinated in their corresponding FMG clinic, and the FMG model-specific vaccine coverage was $67.8 \%$ (95\% CI $60.5 \%-74.4 \%$ ), which did not significantly differ from the baseline CLSC model. The obstetrics model showed significantly lower model-specific vaccine coverage at $35.3 \%$ (95\% CI $29.5 \%-41.5 \%$ ), since only $41.1 \%$ of vaccinated participants recruited to this model actually received their vaccine in that obstetrics clinic. Finally, the OGCT model-specific vaccine coverage was $44.1 \%$ (95\% CI $38.5 \%-49.9 \%)$, which was significantly lower compared with 


\section{Self-reported status in questionnaire $^{*}$}

\section{Immunization Registry}

\section{Medical charts}

\section{Overall vaccine coverage}

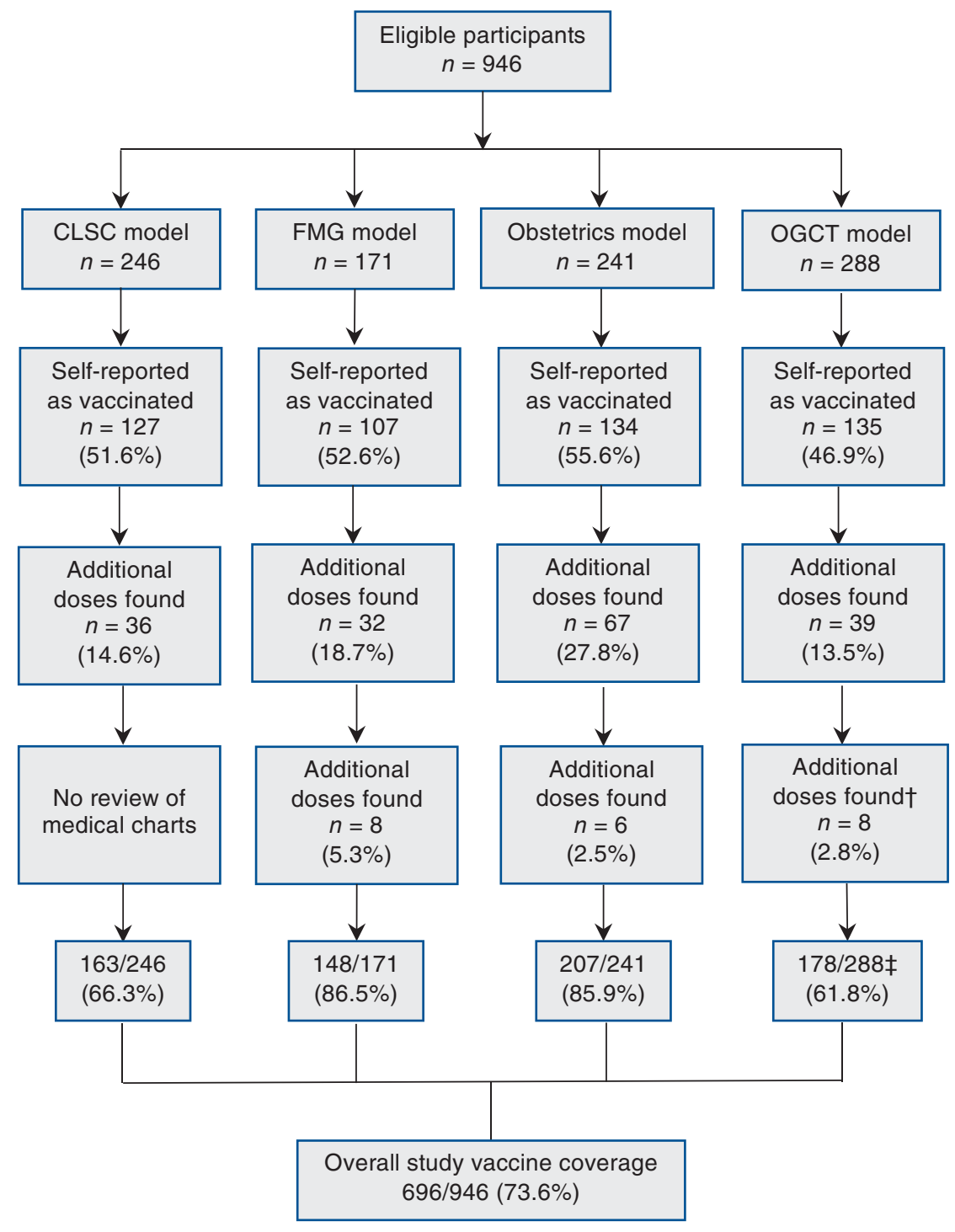

Figure 2: Summary of the process for determining vaccination status using the self-report questionnaire response, the Quebec Immunization Registry and medical charts. Note: CLSC = local community service centre, FMG = family medicine group, OGCT = oral glucose challenge test. ${ }^{*}$ Questionnaire response rate: CLSC model: 159/246 (64.6\%); FMG model: 122/171 (71.3\%); obstetrics model: 142/241 (58.9\%); OGCT model: $196 / 288(68.1 \%)$. †Proof of nonvaccination identified for 4 participants. ‡For the OGCT model, the overall vaccine coverage was adjusted to $178 / 288$ after identifying a proof of nonvaccination for 4 women who self-reported as vaccinated.

the baseline model. The proportion of vaccinated participants recruited to the OGCT model who were vaccinated during their OGCT was $71.4 \%$.

After adjusting for maternal age, country of birth (Canada v. other), education, language and the number of previous children, compared with the CLSC model, the OR of receiving the Tdap vaccine within each vaccine delivery model was 1.26 (95\% CI $0.82-1.94)$ for the FMG model, 0.30 (95\% CI, 0.20-0.44) for the obstetrics model, and 0.47 (95\% CI $0.32-0.68)$ for the OGCT model (Table 2).
Additional sensitivity analyses based on the different assumptions, and vaccine coverage calculated using aggregated data for all participants who presented for their OGCT, regardless of recruitment status (which did not modify the overall vaccine coverage associated with this model) are presented in Appendix 1 (Supplementary Results, Tables S2 and S3).

\section{Overall vaccine coverage}

To compare overall vaccine uptake, we evaluated overall vaccine coverage, considering all doses administered regardless of place of vaccination (Table 2). For the baseline CLSC model, 


\begin{tabular}{|c|c|c|c|c|c|c|c|c|c|}
\hline \multirow[b]{2}{*}{$\begin{array}{l}\text { Vaccine } \\
\text { delivery } \\
\text { model }\end{array}$} & \multirow[b]{2}{*}{$\begin{array}{c}\text { No. of } \\
\text { vaccinated } \\
\text { participants }\end{array}$} & \multirow{2}{*}{$\begin{array}{l}\text { No. }(\%) \text { of } \\
\text { vaccinated } \\
\text { participants } \\
\text { who were } \\
\text { vaccinated } \\
\text { within the } \\
\text { model }\end{array}$} & \multicolumn{3}{|c|}{ Model-specific VC } & \multicolumn{4}{|c|}{ Overall VC } \\
\hline & & & $\begin{array}{l}\text { participants } \\
\text { vaccinated } \\
\text { within the } \\
\text { model } \\
(95 \% \mathrm{Cl})\end{array}$ & $\begin{array}{c}\mathrm{OR}^{*} \\
(95 \% \mathrm{Cl})\end{array}$ & $\begin{array}{c}\text { Adjusted } \\
\text { OR† } \\
(95 \% \mathrm{Cl})\end{array}$ & $\begin{array}{l}\text { Overall no. (\%) } \\
\text { of participants } \\
\text { vaccinated } \\
(95 \% \mathrm{Cl})\end{array}$ & $\begin{array}{l}\text { Absolute } \\
\text { VC } \\
\text { difference, } \\
\quad \%\end{array}$ & $\begin{array}{c}\mathrm{OR}^{*} \\
(95 \% \mathrm{Cl})\end{array}$ & $\begin{array}{c}\text { Adjusted } \\
\text { OR† } \\
(95 \% \mathrm{Cl})\end{array}$ \\
\hline $\begin{array}{l}\text { CLSC, } \\
n=246\end{array}$ & 163 & $\begin{array}{c}157 \\
(96.3)\end{array}$ & $\begin{array}{c}157(63.8) \\
(57.6-69.6)\end{array}$ & $\begin{array}{c}1 \\
\text { (Ref.) }\end{array}$ & $\begin{array}{c}1 \\
\text { (Ref.) }\end{array}$ & $\begin{array}{c}163(66.3) \\
(60.1-71.9)\end{array}$ & Ref. & $\begin{array}{c}1 \\
\text { (Ref.) }\end{array}$ & $\begin{array}{c}1 \\
\text { (Ref.) }\end{array}$ \\
\hline $\begin{array}{l}\text { FMG, } \\
n=171\end{array}$ & 148 & $\begin{array}{c}116 \\
(78.4)\end{array}$ & $\begin{array}{c}116(67.8) \\
(60.5-74.4)\end{array}$ & $\begin{array}{c}1.20 \\
(0.79-1.81)\end{array}$ & $\begin{array}{c}1.26 \\
(0.82-1.94)\end{array}$ & $\begin{array}{c}148(86.5) \\
(80.6-90.9)\end{array}$ & 20.2 & $\begin{array}{c}3.28 \\
(1.99-5.57)\end{array}$ & $\begin{array}{c}4.05 \\
(2.36-7.22)\end{array}$ \\
\hline $\begin{array}{l}\text { Obstetrics, } \\
n=241\end{array}$ & 207 & $\begin{array}{c}85 \\
(41.1) \\
\end{array}$ & $\begin{array}{c}85(35.3) \\
(29.5-41.5) \\
\end{array}$ & $\begin{array}{c}0.31 \\
(0.21-0.45) \\
\end{array}$ & $\begin{array}{c}0.30 \\
(0.20-0.44) \\
\end{array}$ & $\begin{array}{c}207(85.9) \\
(80.9-89.7)\end{array}$ & 19.6 & $\begin{array}{c}3.10 \\
(1.99-4.91)\end{array}$ & $\begin{array}{c}3.37 \\
(2.11-5.49)\end{array}$ \\
\hline $\begin{array}{l}\text { OGCT, } \\
n=288\end{array}$ & 178 & $\begin{array}{c}127 \\
(71.3)\end{array}$ & $\begin{array}{c}127(44.1) \\
(38.5-49.9)\end{array}$ & $\begin{array}{c}0.45 \\
(0.31-0.63)\end{array}$ & $\begin{array}{c}0.47 \\
(0.32-0.68)\end{array}$ & $\begin{array}{c}178(61.8) \\
(56.1-67.2)\end{array}$ & 4.5 & $\begin{array}{c}0.82 \\
(0.58-1.17)\end{array}$ & $\begin{array}{c}0.92 \\
(0.62-1.37)\end{array}$ \\
\hline \multicolumn{10}{|c|}{$\begin{array}{l}\text { Note: } \mathrm{Cl}=\text { confidence interval, } \mathrm{CLSC}=\text { local community service centre, } \mathrm{FMG}=\text { family medicine group, } \mathrm{OGCT}=\text { oral glucose challenge test, } \mathrm{OR}=\text { odds ratio, } \\
\text { Ref. = reference, } \mathrm{VC}=\text { vaccine coverage. } \\
\text { *Odds ratios calculated from univariate logistic regression. } \\
\text { †Adjusted ORs calculated from multivariable logistic regression, adjusting for maternal age, country of birth (Canada v. other), education, language and the number of } \\
\text { previous children. }\end{array}$} \\
\hline
\end{tabular}

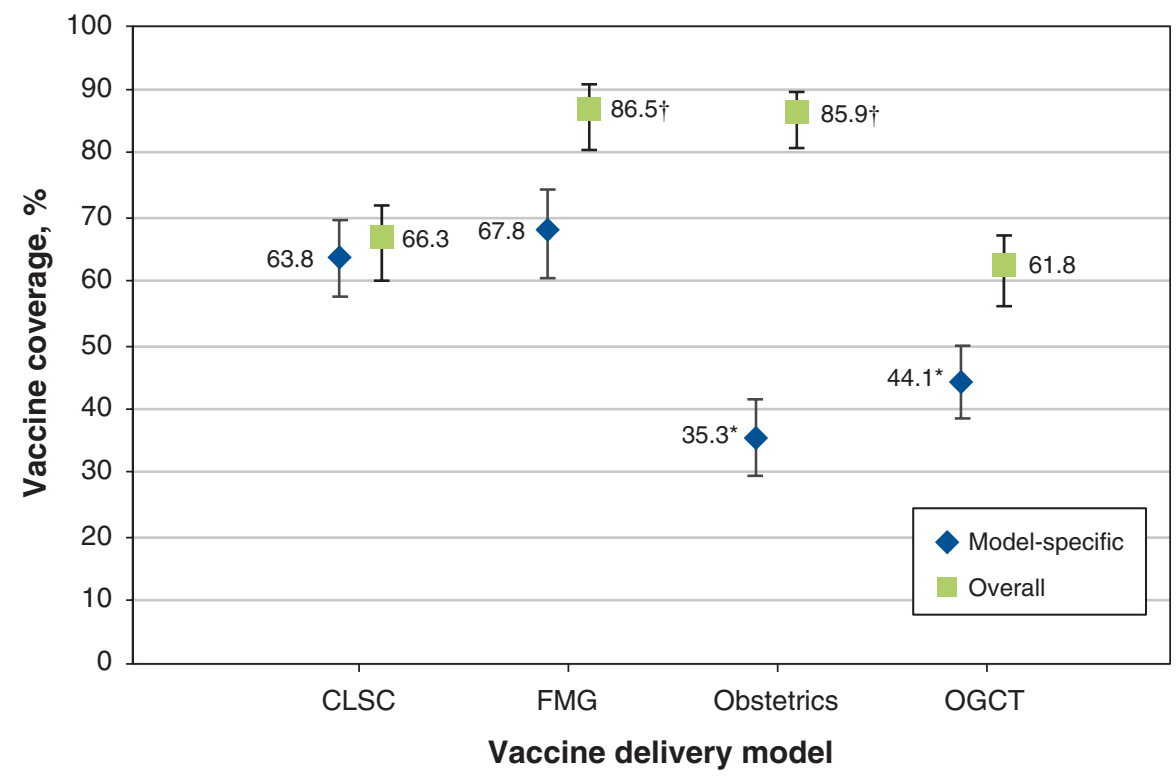

Figure 3: Model-specific and overall vaccine coverage (with 95\% confidence interval) of the 4 vaccine delivery models. Note: CLSC = local community service centre, FMG = family medicine group, OGCT = oral glucose challenge test. *The model-specific vaccine coverage of the obstetrics and OGCT models was significantly lower than the model-specific vaccine coverage of the baseline CLSC model $(p<0.001)$. †The overall vaccine coverage of the FMG and obstetrics models was significantly higher than the overall vaccine coverage of the baseline CLSC model $(p<0.001)$.

overall Tdap vaccine coverage was $66.3 \%$ (95\% CI $60.1 \%-$ $71.9 \%)$. In comparison, a significantly higher vaccine coverage was achieved by the FMG model $(86.5 \%, 95 \%$ CI $80.6 \%-90.9 \%)$ and the obstetrics model $(85.9 \%, 95 \% \mathrm{CI}$ $80.9 \%-89.7 \%)$. However, the OGCT model was not associated with a statistically significant difference in overall vaccine coverage compared with the baseline model $(61.8 \%, 95 \%$ CI
56.1\%-67.2\%). For the FMG, obstetrics and OGCT models, most of the vaccines given outside of the delivery models took place in CLSCs (Appendix 1, Table S4).

Overall, maternal age above the median age of 31 years $(p=0.008)$ and nulliparity $(p<0.001)$ were significantly associated with higher likelihood of vaccination. Being born in Canada $(p=0.070)$, having completed university-level 
education $(p=0.098)$ and being Francophone $(p=0.071)$ were positively associated with Tdap vaccination without these associations being significant.

The overall adjusted OR of receiving the Tdap vaccine was 4.05 for the FMG model (95\% CI 2.36-7.22), 3.37 for the obstetrics model (95\% CI 2.11-5.49) and 0.92 for the OGCT model (95\% CI 0.62-1.37), compared with the CLSC model (Table 2).

\section{Interpretation}

Referring patients to public health clinics (i.e., the CLSC model) is the current standard model for maternal Tdap vaccination in Quebec. Under this model, model-specific and overall vaccine coverage was moderately good, but not optimal (63.8\% and $66.3 \%$, respectively). The CLSC model was highly used by participants and thus resulted in similar or higher model-specific vaccine coverage compared with other delivery models. Nevertheless, the FMG and obstetrics models achieved significantly higher overall vaccine coverage ( $86.5 \%$ and $85.9 \%$, respectively), a difference of about 20 percentage points compared with the CLSC model.

A previous study ${ }^{21}$ reported that vaccination would be facilitated if offered in FMG clinics where pregnancies are followed. In our study, model-specific vaccine coverage was similar for the FMG and the CLSC models (about 65\%), but overall vaccine coverage increased to $85 \%$ for the FMG model when considering all vaccination venues. Participants were more likely to receive Tdap when it was recommended and offered by their family physician, which could be explained by a stronger perceived importance of vaccination from the family physician or nurse offering prenatal care. High maternal Tdap vaccine coverage was also observed in studies from Belgium and the $\mathrm{UK}$, where the main vaccine administrators were general practitioners. ${ }^{12,13}$ In our context, the high vaccine coverage of the FMG model suggested potential benefits of integrating vaccination into prenatal care, as offered in FMG clinics.

The obstetrics clinic offering Tdap vaccination during pregnancy follow-up also achieved significantly higher overall vaccine coverage than the CLSC model. A vaccine offer within the clinic may increase accessibility to Tdap vaccination and emphasize its importance. Maternal pertussis immunization recommendations from health care professionals traditionally involved in pregnancy, including obstetricians, have been associated with higher vaccine coverage. ${ }^{9,13}$ Interestingly, within our obstetrics model, $41 \%$ of vaccinated participants received their vaccine at the obstetrics clinic. It was likely that participants decided to be vaccinated at their CLSC for reasons such as geographical accessibility or limited appointment availability; the nurse offering Tdap vaccination at the obstetrics clinic worked 2 days per week. Results could be different with another obstetric clinic organization.

The impact of offering Tdap vaccination at the time of gestational diabetes screening had not been well studied. Our study showed that this strategy did not improve model-specific or overall vaccine coverage. Suboptimal model-specific vaccine coverage could be explained in part by the timing of the vaccine offer (before 26 weeks' gestation) or because the OGCT was perceived as an ordeal in itself. Participants with diabetes or prediabetes who were not present for an OGCT appointment were referred to a CLSC, although the exclusion of these participants from a sensitivity analysis did not improve the model-specific vaccine coverage. Nevertheless, participants used the OGCT model more than the obstetrics clinic, as a higher percentage $(71.4 \%)$ of vaccinated participants received their vaccine during their OGCT. This may highlight benefits of opportunistic vaccination services at the OGCT appointment, which overlaps with the ideal time for maternal pertussis immunization. ${ }^{21-23}$ Future research is needed to evaluate strategies combining physician or obstetrician referral for OGCT and offering of Tdap during an OGCT appointment. Integration of Tdap vaccination with other prenatal services should also be considered, which could refer pregnant people to other resources if they miss this vaccination opportunity.

\section{Limitations}

In the present study, strengths include the quasiexperimental study design, which is effective at evaluating vaccine delivery interventions, ${ }^{26}$ and the validation of vaccination history. The participation rate was lower for the obstetrics model, which could lead to a slight overestimation of vaccine coverage if nonparticipating pregnant people were less likely to be vaccinated. Reassuringly, the participation rate was high (96\%) for 1 of the recruitment sites, and very high vaccine coverage was also obtained. We did not expect an important selection bias in terms of vaccine acceptance for those who refused to participate. We did not review medical charts for the CLSC model, but after a sensitivity analysis addressing this issue, conclusions remained similar to the primary analysis. Participants differed in sociodemographic characteristics at baseline, which might have influenced their likelihood of Tdap vaccination. We accounted for these differences in our multivariable models. Finally, our sample recruited for the OGCT model may not be representative of the population of those who access prenatal care services in the province or the country, since the hospital where this model was implemented serves a large urban multiethnic catchment area and is the referral centre for high-risk pregnancies.

\section{Conclusion}

Compared with CLSCs, overall vaccine coverage was higher when Tdap was offered in FMGs or an obstetrics clinic providing prenatal care. Recommendations and offers of vaccination from health professionals involved in routine pregnancy follow-up may be the key factors in optimizing vaccine coverage. Our findings and additional analyses on vaccine acceptability, as well as on costs of vaccination strategies, will be important to understand the successful implementation of universal maternal pertussis vaccination in Quebec and elsewhere. 


\section{References}

1. Brophy J, Baclic O, Tunis MC. Summary of the NACI update on immunization in pregnancy with tetanus toxoid, reduced diphtheria toxoid and reduced acellular pertussis (Tdap) vaccine. Can Commun Dis Rep 2018;44:91-4.

2. Abu-Raya B, Bettinger JA, Vanderkooi OG, et al. Burden of children hospitalized with pertussis in Canada in the acellular pertussis vaccine era, 1999-2015. 7 Pediatric Infect Dis Soc 2020;9:118-27.

3. Pertussis vaccine: Canadian Immunization Guide. Ottawa: Public Health Agency of Canada; modified 2021 Sept. 23. Available: https://www.canada.ca/en/ public-health/services/publications/healthy-living/canadian-immunization-guide -part-4-active-vaccines/page-15-pertussis-vaccine.html (accessed 2020 Aug. 10).

4. Fleurant-Ceelen A, Tunis M, House A. What is new in the Canadian Immunization Guide: November 2016 to November 2018. Can Commun Dis Rep 2018;44:331-5.

5. Abu Raya B, Edwards KM, Scheifele DW, et al. Pertussis and influenza immunisation during pregnancy: a landscape review. Lancet Infect Dis 2017; 17:e209-22.

6. Whooping cough. Gouvernement du Québec. Available: https://www.quebec.ca/ en/health/health-issues/a-z/whooping-cough/\#c6954 (accessed 2020 Aug. 18)

7. Koepke R, Kahn D, Petit AB, et al. Pertussis and influenza vaccination among insured pregnant women: Wisconsin, 2013-2014. MMWR Morb Mortal Wkly Rep 2015;64:746-50.

8. Barber A, Muscoplat MH, Fedorowicz A. Coverage with tetanus, diphtheria, and acellular pertussis vaccine and influenza vaccine among pregnant women: Minnesota, March 2013-December 2014. MMWR Morb Mortal Wkly Rep 2017;66:56-9.

9. Kerr S, Van Bennekom CM, Liang JL, et al. Tdap vaccination coverage during pregnancy: selected sites, United States, 2006-2015. MMWR Morb Mortal Wkly Rep 2017;66:1105-8.

10. Ghaswalla P, Poirrier J-E, Packnett ER, et al. Maternal immunization in the U.S.: a nationwide retrospective cohort study. Am 7 Prev Med 2019;57:e87-93.

11. Razzaghi H, Kahn KE, Black CL, et al. Influenza and Tdap vaccination coverage among pregnant women: United States, April 2020. MMWR Morb Mortal Wkly Rep 2020;69:1391-7.

12. Pertussis vaccination programme for pregnant women: vaccine coverage in England, January to March 2020 and 2019-20 annual coverage. Health Protection Report, Volume 14, Number 10. London (UK): Public Health England; 2020:1-9. Available: https://assets.publishing.service.gov.uk/ government/uploads/system/uploads/attachment_data/file/887216/hpr1020_ prtsss-vc.pdf (accessed 2020 Aug. 10).

13. Maertens K, Braeckman T, Top G, et al. Maternal pertussis and influenza immunization coverage and attitude of health care workers towards these recommendations in Flanders, Belgium. Vaccine 2016;34:5785-91.

14. Laenen J, Roelants M, Devlieger R, et al. Influenza and pertussis vaccination coverage in pregnant women. Vaccine 2015;33:2125-31.

15. Griffin JB, Yu L, Watson D, et al. Pertussis immunisation in pregnancy safety (PIPS) study: a retrospective cohort study of safety outcomes in pregnant women vaccinated with Tdap vaccine. Vaccine 2018;36:5173-9.

16. Deverall EJ, Gilmore B, Illing S, et al. Pertussis vaccination uptake in pregnancy: lessons to be learned from an integrated healthcare approach. $N Z \mathrm{Med}$ 7 2018;131:42-7.

17. Erb ML, Erlanger TE, Heininger U. Child-parent immunization survey: How well are national immunization recommendations accepted by the target groups? Vaccine X 2019;1:100013.

18. Amirthalingam G, Andrews N, Campbell H, et al. Effectiveness of maternal pertussis vaccination in England: an observational study. Lancet 2014;384:1521-8.

19. Brillo E, Tosto V, Giardina I, et al. Maternal tetanus, diphtheria, and acellular pertussis (Tdap) and influenza immunization: an overview. 7 Matern Fetal Neonatal Med 2021;34:3415-44.

20. Ahluwalia IB, Ding H, D'Angelo D, et al.; Centers for Disease Control and Prevention (CDC). Tetanus, diphtheria, pertussis vaccination coverage before, during, and after pregnancy: 16 states and New York City, 2011. MMWR Morb Mortal Wkly Rep 2015;64:522-6.

21. Brousseau N, Gagnon D, Vivion M, et al. Expected challenges of implementing universal pertussis vaccination during pregnancy in Quebec: a crosssectional survey. CMA7 Open 2018;6:E391-7.

22. An Advisory Committee Statement (ACS) National Advisory Committee on Immunization (NACI): update on immunization in pregnancy with tetanus toxoid, reduced diphtheria toxoid and reduced acellular pertussis (Tdap) vaccine. Ottawa: Public Health Agency of Canada; 2018, modified 2019 Oct. 9. Available: https://www.canada.ca/en/public-health/services/publications/ healthy-living/update-immunization-pregnancy-tdap-vaccine.html (accessed 2020 Aug. 10)

23. Committee Opinion No. 718: Update on immunization and pregnancy — tet anus, diphtheria, and pertussis vaccination. Obstet Gynecol 2017;130:e153-7.

24. Institut de la statistique du Québec. Population estimates for administrative regions, Québec, July 1, 1986 to 2020 (in French only). Gouvernement du Québec; updated 2021 Jan. 14. Available: https://statistique.quebec.ca/en/ produit/tableau/estimations-population-regions-administratives (accessed 2021 July 1).

25. Immioration Francisation et Intéoration Québec. Where to settle? Gouvernement du Québec; updated 2021 May 17. Available: https://www.immigration -quebec.gouv.qc.ca/en/settle/index.html (accessed 2021 July 1).

26. Lopez Bernal JA, Andrews N, Amirthalingam G. The use of quasi-experimental designs for vaccine evaluation. Clin Infect Dis 2019;68:1769-76.

Affiliations: Department of Epidemiology, Biostatistics \& Occupational Health (Li), McGill University; CHU Sainte-Justine Research Center (Li, Laghdir, Boucoiran, Tapiéro), Montréal, Que.; Direction des risques biologiques et de la santé au travail (Brousseau, Dubé), Institut national de la santé publique du Québec; CHU de Quebec Research Center (Brousseau, Dubé), Université Laval, Québec City, Que.; Research Center (Guay), Hôpital Charles-Lemoyne, Longueuil, Que.; Département des sciences de la santé communautaire (Guay), Université de Sherbrooke, Sherbrooke, Que.; Department of Obstetrics \& Gynecology (Boucoiran), School of Public Health, Université de Montréal; Infectious Diseases Division (Tapiéro), Department of Pediatrics, CHU SainteJustine; Department of Microbiology, Infectious Diseases and Immunology (Quach), Université de Montréal; Infection Prevention and Control (Quach), Clinical Department of Laboratory Medicine, CHU SainteJustine, Montréal, Que.

Contributors: Nicholas Brousseau, Maryse Guay, Ève Dubé, Isabelle Boucoiran, Bruce Tapiéro and Caroline Quach conceived the study, and Yinan Li, Nicholas Brousseau, Maryse Guay, Evve Dubé, Isabelle Boucoiran, Bruce Tapiéro and Caroline Quach contributed to the study design. Zineb Laghdir drafted the study protocol. All of the authors contributed to data analysis and interpretation, and to drafting of the manuscript. All of the authors critically revised the manuscript for important intellectual content, gave final approval of the version to be published and agreed to be accountable for all aspects of the work.

Funding: This work was funded by the Quebec Ministry of Health and Social Services (Ministère de la Santé et des Services sociaux).

Content licence: This is an Open Access article distributed in accordance with the terms of the Creative Commons Attribution (CC BY-NC-ND 4.0) licence, which permits use, distribution and reproduction in any medium, provided that the original publication is properly cited, the use is noncommercial (i.e., research or educational use), and no modifications or adaptations are made. See: https://creativecommons.org/licenses/by-nc-nd/4.0/

Data sharing: Data presented in this manuscript are available after project completion upon request to other investigators who do not have access to public servers in the province of Quebec.

Acknowledgements: The authors thank Maryline Vivion, Marie-Claude Gariépy, Josiane Rivard and all those who contributed to data collection. They also thank the study participants and regional collaborators who made this project possible.

Supplemental information: For reviewer comments and the original submission of this manuscript, please see www.cmajopen.ca/content /10/1/E56/suppl/DC1. 